\title{
La piel: un testigo silencioso en la esclerosis sistémica
}

The skin: A silent witness in systemic sclerosis

\section{Claudia Marcela Arenas', Paola Andrea Olaya ${ }^{2}$}

1. Médica dermatóloga, especialista en Docencia Universitaria, Centro Dermatológico Federico Lleras Acosta, Bogotá, D.C., Colombia.

2. Médica, residente de tercer año de Dermatología, Fundación Universitaria Sanitas-Centro Dermatológico Federico Lleras Acosta, Bogotá, D.C., Colombia.

\section{RESUMEN}

Son varios los hallazgos cutáneos que sugieren al dermatólogo el diagnóstico de esclerosis sistémica; entre ellos, se describen: disminución de la apertura oral, pérdida de arrugas faciales, fenómeno de Raynaud, megacutícula, esclerodactilia, calcificaciones y cambios en la pigmentación de la piel, esclerosis cutánea y alteraciones en la capilaroscopia. Muchos de estos hallazgos dermatológicos nos permiten orientar el diagnóstico, clasificar la enfermedad, indicar un tratamiento oportuno y evaluar el pronóstico, por lo cual es importante conocerlos.

Se revisan los hallazgos dermatológicos en pacientes con esclerosis sistémica.

PALABRAS CLAVE: esclerosis, tejido conjuntivo, esclerodermia, esclerodermia limitada, esclerodermia difusa.

\section{SUMMARY}

There are various cutaneous findings that suggest the diagnosis of systemic sclerosis to the dermatologist; among them are described the limitation of mouth opening, reduction of facial wrinkles, Raynaud's phenomenon, sclerodactyly, nailfold capillaroscopy changes, calcifications and changes in the skin pigmentation and cutaneous sclerosis. It is important to know many of these dermatological findings since they allow us to guide the diagnosis, staging the disease, indicating early treatment and assess the prognosis.

In this article we review the dermatological findings in patients with systemic sclerosis.

KEY WORDS: Sclerosis, connective tissue, systemic scleroderma, limited scleroderma, diffuse scleroderma.

\section{INTRODUCCIÓN}

La esclerosis sistémica es una enfermedad crónica, multisistémica, de curso clínico variable, con pobre pronóstico y con las mayores tasas de morbimortalidad entre las enfermedades reumáticas autoinmunitarias.

Se caracteriza por fibrosis cutánea, alteraciones vasculares y autoanticuerpos contra varios antígenos celulares ${ }^{1-4}$.
Correspondencia:

Claudia Marcela Arenas

Email:

Draclaudiaarenas@gmail.com

Recibido: 25 de marzo de 2015

Aceptado: 17 de junio de 2016

No se reportan conflictos de interés. 


\begin{tabular}{|c|c|c|}
\hline CATEGORÍA & SUBÍTEM & SCORE \\
\hline Piel & $\begin{array}{l}\text { - } \quad \text { Engrosamiento de la piel de los dedos de ambas manos } \\
\text { - } \quad \text { Dedos edematosos } \\
\text { - Esclerodactilia }\end{array}$ & $\begin{array}{l}9 \\
2 \\
4\end{array}$ \\
\hline Lesiones en pulpejo de dedos & $\begin{array}{l}\text { - Úlceras digitales } \\
\text { - } \quad \text { Cicatrices en punta de los dedos }\end{array}$ & $\begin{array}{l}2 \\
3\end{array}$ \\
\hline Telangiectasias & -- & 2 \\
\hline Capilaroscopia anormal & -- & 2 \\
\hline $\begin{array}{l}\text { Hipertensión pulmonar y/o enfermedad } \\
\text { pulmonar intersticial }\end{array}$ & -- & 2 \\
\hline Fenómeno de Raynaud & -- & 3 \\
\hline $\begin{array}{l}\text { Anticuerpos (anti centrómero, anti- } \\
\text { topoisomerasa-I (anti-Scl-70), anti- RNA } \\
\text { polimerasa -3) }\end{array}$ & $\cdots$ & 3 \\
\hline
\end{tabular}

TABLA 1. Criterios diagnósticos en la clasificación de la esclerosis sistémica ${ }^{11}$.

La piel es un marcador de la seriedad y del pronóstico en la esclerosis sistémica. Los signos cutáneos son unas de sus primeras manifestaciones; de ahí, la importancia de identificarlos para hacer un diagnóstico temprano y brindar un tratamiento oportuno que permita modificar el curso natural de la enfermedad.

\section{EPIDEMIOLOGÍA}

La esclerosis sistémica es una enfermedad poco frecuente, con una prevalencia de 1 a 15 casos por 100.000 habitantes y una incidencia anual de 0,3 a 2,8 casos por 100.000 habitantes en los Estados Unidos. La edad media de aparición es entre los 30 y 50 años, y la supervivencia media desde el diagnóstico es de 12 años $^{5-7}$.

Esta entidad afecta a mujeres y a hombres con una proporción de 3:1; es más frecuente en personas de raza negra e infrecuente en los niños ${ }^{2,8-10}$.

\section{CLASIFICACIÓN}

La esclerosis sistémica tiene dos subtipos principales: la cutánea limitada y la cutánea difusa.

Para la clasificación de la esclerosis sistémica existen criterios diagnósticos actualizados por el Colegio Americano de Reumatología (ACR) y la Liga Europea Contra el Reumatismo (EULAR) en el año 2013, un puntaje de 9 o más es diagnóstico de esclerosis sis- témica ${ }^{11}$ (TABLA 1). En otras formas de presentación de la esclerosis sistémica, como en aquella sin esclerodermia, en los estados iniciales de la enfermedad y en la pre-esclerodermia, estos criterios no son útiles ${ }^{12,13}$ (TABLA 2).

\section{ETIOLOGÍA}

La esclerosis sistémica es una enfermedad multifactorial en la que no se ha identificado un antígeno específico desencadenante; sin embargo, se considera que un estímulo ambiental o infeccioso en un individuo predispuesto genéticamente activa al sistema inmunológico $^{14,15}$. Esta activación estimula la liberación de citocinas proinflamatorias, como IL-1 $\beta$, IL-2, IL-4, IL-6, de factor de necrosis tumoral $\alpha$, de factor de crecimiento transformante $\beta$, de endotelina-1 y de autoanticuerpos, como antitopoisomerasa-I (SCl-70), anti-ARN polimerasa III y anticentrómero, que inician, mantienen y amplifican la reacción inmunógica y, en general, inducen disfunción endotelial, proliferación de fibroblastos y síntesis de colágeno $0^{5,16,17}$.

\section{HALLAZGOS DERMATOLÓGICOS Fenómeno de Raynaud}

Corresponde al vasoespasmo arteriolar paroxístico como reacción a un estímulo emocional o por frío ${ }^{18,19}$. Ocurre 


\begin{tabular}{|c|c|c|c|c|}
\hline Hallazgos clínicos & $\begin{array}{l}\text { Esclerosis sistémica } \\
\text { cutánea limitada }\end{array}$ & $\begin{array}{l}\text { Esclerosis sistémica } \\
\text { cutánea difusa }\end{array}$ & $\begin{array}{l}\text { Esclerosis sistémica } \\
\text { sin esclerodermia }\end{array}$ & Pre- esclerodermia \\
\hline Fenómeno de Raynaud & $\begin{array}{l}\text { Precede en años a la } \\
\text { aparición de la clínica } \\
\text { cutánea }\end{array}$ & $\begin{array}{l}\text { De pocos meses de } \\
\text { duración e incluso } \\
\text { posterior a la aparición } \\
\text { de la clínica cutánea }\end{array}$ & Sí & Sí \\
\hline $\begin{array}{l}\text { Localización de la } \\
\text { esclerosis }\end{array}$ & $\begin{array}{l}\text { Limitada a la cara y } \\
\text { zonas distales (acral) }\end{array}$ & $\begin{array}{l}\text { Tronco y parte proximal } \\
\text { de las extremidades }\end{array}$ & Sin esclerosis & Sin esclerosis \\
\hline Cambios capilares & $\begin{array}{c}\text { Capilares periungulares } \\
\text { dilatados }\end{array}$ & $\begin{array}{l}\text { Dilatación capilar y } \\
\text { pérdida de capilares } \\
\text { periungulares }\end{array}$ & $\begin{array}{l}\text { Capilaroscopia } \\
\text { anormal }\end{array}$ & $\begin{array}{l}\text { Cambios en la } \\
\text { capilaroscopia } \\
\text { periungular y } \\
\text { evidencia de } \\
\text { isquemia digital }\end{array}$ \\
\hline Úlceras digitales & Sí & $\begin{array}{l}\text { Sí, con mayor } \\
\text { frecuencia }\end{array}$ & Sí & Sí \\
\hline Pulmonares & $\begin{array}{l}\text { Hipertensión arterial } \\
\text { pulmonar tardía } \\
\quad(10-15 \%) \text { con } \\
\text { enfermedad intersticial } \\
\text { pulmonar o sin ella. }\end{array}$ & $\begin{array}{l}\text { Compromiso } \\
\text { pulmonar intersticial } \\
\text { con fibrosis } \\
\text { Hipertensión } \\
\text { pulmonar secundaria }\end{array}$ & \multicolumn{2}{|l|}{$\begin{array}{l}\text { Hipertensión arterial } \\
\text { pulmonar (sin } \\
\text { fibrosis) }\end{array}$} \\
\hline $\begin{array}{l}\text { Calcificaciones } \\
\text { cutáneas }\end{array}$ & $\begin{array}{l}\text { Sí, con mayor } \\
\text { frecuencia }\end{array}$ & Sí & No & No \\
\hline Gastrointestinales & $\begin{array}{c}\text { Con compromiso o } \\
\text { sin él }\end{array}$ & Compromiso difuso & $\begin{array}{l}\text { Hipomotilidad } \\
\text { esofágica distal y del } \\
\text { intestino delgado }\end{array}$ & No \\
\hline Renales & Raro & $\begin{array}{l}\text { Temprano y } \\
\text { significativo }\end{array}$ & Crisis renal & \\
\hline Cardíacos & $\begin{array}{c}\text { Con compromiso o } \\
\text { sin él }\end{array}$ & $\begin{array}{l}\text { Compromiso } \\
\text { miocárdico }\end{array}$ & $\begin{array}{c}\text { Afectación cardíaca } \\
\text { típica }\end{array}$ & \\
\hline \multicolumn{5}{|c|}{ Perfil inmunológico } \\
\hline ACA & $60 \%$ & $2 \%$ & \multirow{4}{*}{$\begin{array}{c}\text { ANA positivos } \\
\text { (antitopoisomerasa-I, } \\
\text { ACA o anti } \\
\text { RNA-polimerasa I, II } \\
\text { o III) }\end{array}$} & \multirow{4}{*}{$\begin{array}{l}\text { Autoanticuerpos } \\
\text { específicos } \\
\text { circulantes } \\
\text { antitopoisomeras } \\
\text { - I (scl-70), } \\
\text { ACA o anti RNA } \\
\text { polimerasa I, II o III }\end{array}$} \\
\hline Anti Scl- 70 & $15 \%$ & $40 \%$ & & \\
\hline Anti-RNA polimerasa III & - & $40 \%$ & & \\
\hline Anti PM-Scl & $2 \%$ & $25 \%$ & & \\
\hline
\end{tabular}

TABLA 2. Clasificación de la esclerosis sistémica.

en el $95 \%$ de los pacientes con esclerosis sistémica y en menos del $10 \%$ de la población general ${ }^{20}$. Se debe a tres mecanismos patogénicos: vasoconstricción, isquemia y reperfusión. El fenómeno de Raynaud se caracteriza por palidez brusca seguida de cianosis y, a veces, de eritema doloroso secundario a reperfusión vascular. Por lo general, afecta zonas distales $(\text { acral })^{2,5,20,21}$ y puede aparecer meses e incluso años antes del desarrollo de la esclerosis de la piel. La hipoperfusión intermitente causa necrosis en los pulpejos y pliegues ungulares, que habitualmente conduce a ulceraciones y formación de cicatrices, con pérdida de tejido de los pulpejos y reducción gradual de su tamaño, lo cual resulta en atrofia digital 2,22-24. Otros cambios cutáneos que se pueden observar en la esclerosis sistémica son onicólisis, onicodistrofía y megacutícula 5 (FIGURA 1A). 

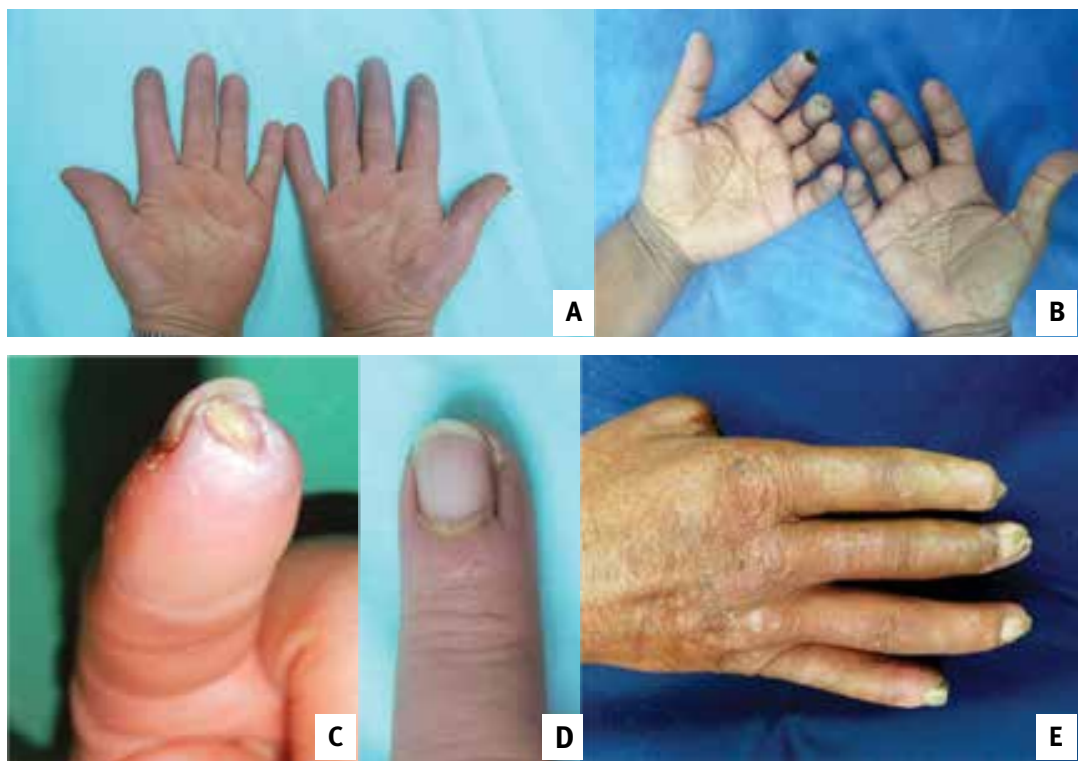

FIGURA 1. Hallazgos dermatológicos en pacientes con esclerosis sistémica. A. Fenómeno de Raynaud donde se observa cianosis distal. B. Necrosis y cicatrices digitales secundarias a la enfermedad microvascular. C. Úlcera digital. D. Megacutícula. E.

Acortamiento de las falanges distales y deformidad ungular como resultado de la resorción ósea. Amputación digital y también se observa esclerodactilia.
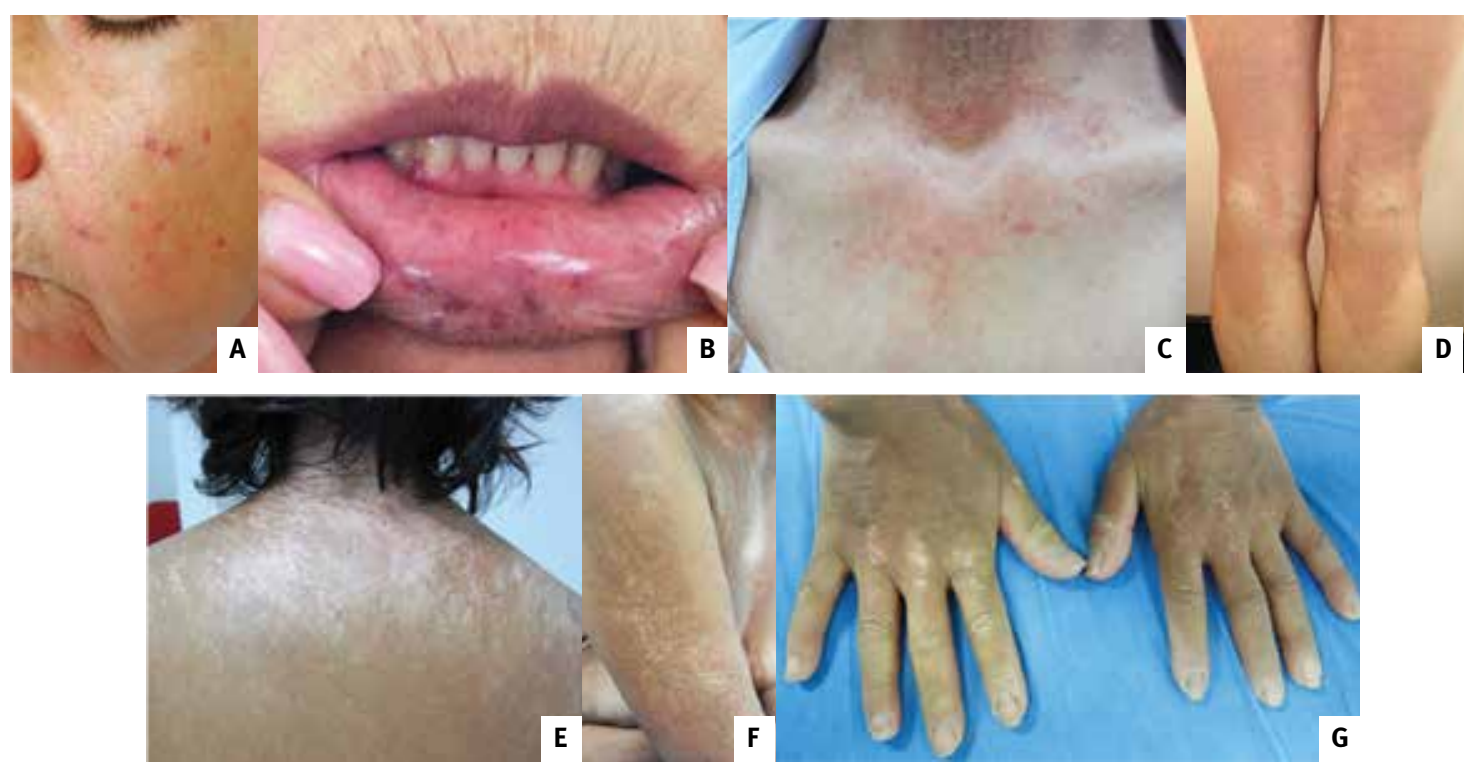

FIGURA 2. Hallazgos dermatológicos en pacientes con esclerosis sistémica. A, B y C. En esta paciente se observan telangiectasias en mejillas, $\mathrm{V}$ del escote y mucosa labial, características del anteriormente denominado síndrome CREST (calcinosis, Raynaud phenomenon, esophageal dismotility, sclerodactily and telangiectasia). Estas telangiectasias pueden blanquearse por la presión. Pueden ser confundidas con telangiectasia hemorrágica hereditaria (enfermedad de Osler-Weber-Rendu). D. Placas atróficas hiperpigmentadas. E y F. Paciente con esclerosis sistémica cutánea difusa. Se observan placas escleróticas con hiperpigmentación e hipopigmentación que dan el aspecto de sal y pimienta. G. Máculas acrómicas que dan un aspecto similar al vitiligo. 


\section{Úlceras digitales}

Aparecen aproximadamente en el $58 \%$ de los pacientes, $32 \%$ son crónicas y $25 \%$ pueden ser múltiples ${ }^{20,25}$. Su etiología es multifactorial por microtrauma repetido, adelgazamiento cutáneo, piel seca y calcinosis subyacente, pero, el factor principal es la isquemia digital prolongada como expresión del fenómeno de Raynaud, que produce fibroproliferación de la íntima y trombosis de las arterias digitales ${ }^{20,21,26}$. Se localizan principalmente en los pulpejos de los dedos de las manos, denominadas "úlceras por mordedura de rata", y también, en las articulaciones interfalángicas ${ }^{27}$. Son lesiones muy dolorosas y de difícil curación ${ }^{5,20,21,28}$ (FIGURA 1C). Además, se han descrito las denominadas úlceras no digitales, que se localizan en la región pretibial, los tobillos, los codos y el dorso de los pies. Las úlceras pueden ocasionar dolor, sobreinfección, osteomielitis, gangrena, resorción digital y autoamputación, produciendo discapacidad transitoria o permanente. Son más frecuentes en casos de esclerosis sistémica cutánea difusa con afectación cutánea extensa ${ }^{2}$ (FIGURA 1E).

\section{Telangiectasias}

Son otro signo de vasculopatía caracterizado por dilataciones capilares usualmente redondeadas, que se encuentran principalmente en la cara, la V del escote, las manos y los labios (FIGURA 2, A-C). Estos cambios son más frecuentes en la esclerosis sistémica cutánea limitada $a^{2,5,23}$.

Cambios en la pigmentación de la piel. En la esclerosis sistémica hay cambios en la coloración de la piel con áreas de hiperpigmentación o áreas de hiperpigmentación e hipopigmentación que dan el aspecto de sal y pimienta, y otras similares a lesiones de vitiligo ${ }^{5}$ (FIGURA 2, D, E, F, G). El mecanismo de la pigmentación difusa es desconocido. Algunos autores proponen que la IL-1 se produce en exceso en pacientes con esclerosis sistémica, lo que promueve la síntesis de endotelina 1 por parte de los queratinocitos, lo cual estimula la metalogénesis ${ }^{16,29}$.

Calcinosis. Son depósitos de calcio que se localizan en la piel o en el tejido subcutáneo ${ }^{20}$. El depósito se produce sobre todo en los pulpejos, en las articulaciones interfalángicas proximales, las superficies extensoras de codos, las rodillas y en las zonas con traumatismos por presión (FIGURA 3, A-D). Se presentan como nódulos de tamaño variable, que se pueden manifestar con inflamación local, ulceración o sobreinfección, y pueden resolverse al drenarse el material calcificado después de un daño en la piel ${ }^{5,16}$.

Los pacientes con esclerosis sistémica cutánea limitada desarrollan con más frecuencia calcinosis distrófica, la cual se produce por la hipoxia de los tejidos comprometidos, pero, con un metabolismo fosfocálcico normal. La disminución de la perfusión se acompaña de actividad inflamatoria celular con activación de los macrófagos y desequilibrio entre diversos mediadores que producen un aumento del ingreso de calcio en las células ${ }^{20,30,31}$.

Fibrosis cutánea. Es el hallazgo cardinal de la esclerosis sistémica y frecuentemente se inicia en los dedos de las manos. La fibrosis cutánea se produce en tres fases: edematosa, indurada y atrófica. Se inicia con edema sin fóvea y tirantez de intensidad variable en los dedos y en las manos, que es mayor en las mañanas; normalmente es indoloro y produce la apariencia de "dedos en salchicha” (FIGURA 3E). Este cambio aparece temprano durante la enfermedad, usualmente se inicia en la parte distal de las extremidades y avanza proximalmente $e^{2,5,23}$. Tras un tiempo variable (más prolongado en la esclerosis sistémica cutánea limitada), la piel se endurece, se torna brillante, tirante y fuertemente adherida a los tejidos subcutáneos. Los dedos toman una apariencia de huso, llamados "dedos de madonna", que son puntiagudos, con la piel casi inamovible por su fijación al hueso subyacente ${ }^{2,5,23}$ (FIGURA 3F).

Otras manifestaciones. Los pacientes también pueden presentar una apariencia facial en máscara por pérdida de las líneas de expresión, con nariz afilada, microstomía, disminución de la apertura oral con surcos radiales peribucales que dan la apariencia de boca en "bolsa de tabaco" y movilidad limitada de la lengua por esclerosis del frenillo lingual ${ }^{2,5,23}$ (FIGURA 3G). Otros hallazgos descritos son alopecia, anhidrosis y xerosis mucocutánea, que se producen por la pérdida de los anexos cutáneos (folículos pilosos, glándulas sudoriparas y sebáceas $)^{2,5,23}$.

El prurito se presenta por la piel seca, usualmente aparece en etapas iniciales de la esclerosis sistémica cutánea difusa y puede ser muy intenso, pero tiende a mejorar con el tiempo ${ }^{32}$.

\section{MANIFESTACIONES MÚSCULO- ESQUELÉTICAS}

Los síntomas más frecuentes son debilidad muscular, artralgias y artritis. También, se puede encontrar in- 

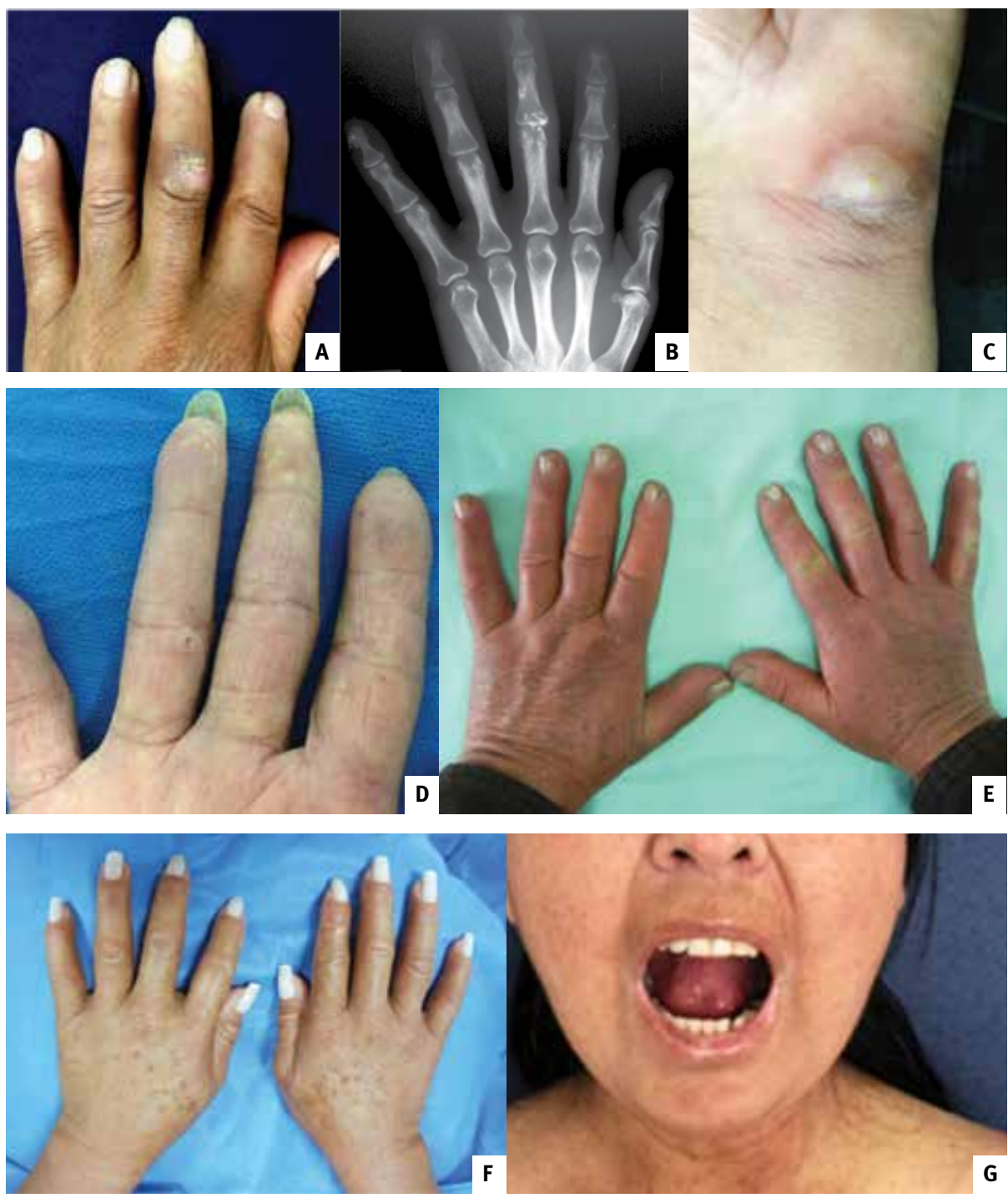

FIGURA 3. Hallazgos dermatológicos en pacientes con esclerosis sistémica. A-D. Paciente con esclerosis sistémica cutánea limitada con calcinosis cutánea en los pulpejos y en las articulaciones interfalángicas proximales de dedos de manos. La calcinosis puede estar asociada con el síndrome CREST. E. Edema sin fóvea y tirantez en los dedos y en las manos que da la apariencia de "dedos en salchicha”. F. Tras un tiempo variable, la piel se endurece y se torna brillante, tirante. Los dedos toman una apariencia de huso, llamados "dedos de madonna”. G. Esta paciente tiene características de esclerosis sistémica, incluyendo piel brillante en las mejillas y nariz, con disminución de líneas de expresión y de la apertura oral que le da apariencia facial en máscara.

movilidad y contracturas articulares que se deben a la fibrosis alrededor de los tendones y otras estructuras periarticulares. Puede aparecer miopatía, con debilidad muscular proximal leve y usualmente no progresiva, miositis y superposición con polimiositis, aunque es poco frecuente ${ }^{2,33,34}$.

\section{DIAGNÓSTICO}

El diagnóstico se establece principalmente con los hallazgos clínicos descritos y, para identificarlos, es necesario hacer una anamnesis detallada y un examen físico completo. La impresión diagnóstica debe confir- 
marse con los estudios paraclínicos que se describen a continuación ${ }^{2}$.

Biopsia de piel. Muestra fibrosis en la dermis reticular y trabéculas fibrosas subcutáneas. Los haces de colágeno son pálidos, homogéneos y edematosos, y puede haber un infiltrado linfocitario perivascular disperso con dilatación de los capilares. En los estados iniciales se puede observar paniculitis con posterior reemplazo de la grasa subcutánea por tejido conjuntivo fibroso. En los estados más avanzados, desaparecen los anexos y se observa aplanamiento de las crestas interpapilares. En los vasos hay proliferación endotelial y oclusión completa de la luz ${ }^{35}$.

Capilaroscopia. En más del $95 \%$ de los pacientes con esclerosis sistémica, la microangiopatía periférica sigue un típico patrón de esclerodermia, consistente en las fases temprana, activa y tardía ${ }^{36}$.

La diferenciación de estos patrones es de gran importancia clínica, dado que el patrón temprano puede ser detectado muchos años antes de la manifestación clínica completa de la enfermedad, y la progresión a patrones activo y tardío se correlaciona estrechamente con compromiso de órganos internos ${ }^{36,37}$.

La capilaroscopia puede ser útil para predecir el desarrollo de ulceraciones digitales en pacientes con esclerosis sistémica ${ }^{36}$.

El índice de riesgo capilaroscópico de ulceración de la piel es una herramienta creada por Sebastiani, et al., basada en la siguiente fórmula ${ }^{36,38}$ :

$\mathrm{D} \times \mathrm{M} / \mathrm{N}^{2}$

donde $\mathrm{D}$ es el diámetro del asa capilar gigante más grande, $\mathrm{M}$ es el número de asas gigantes y $\mathrm{N}$ es el número de todas las asas.

En un ensayo clínico prospectivo, este índice de riesgo predijo correctamente la formación de nuevas ulceraciones en los siguientes tres meses después de la capilaroscopia, con un valor predictivo positivo de $81 \%$ para un índice mayor de 2,96 , y un valor predictivo negativo de $93 \%$ para un índice menor de $2,96^{36,39}$.

Pruebas serológicas. Los anticuerpos antinucleares (ANA) están presentes en 75 a $90 \%$ de los pacientes con esclerosis sistémica; no son específicos de ningún subtipo de la enfermedad ni determinan un pronóstico².

La esclerosis sistémica cutánea difusa se caracteriza por la presencia de anticuerpos anti Scl 70 (topoisomerasa I) y, la esclerosis sistémica cutánea limitada, por la presencia de anticuerpos anticentrómero (ACA) ${ }^{40-44}$ (TABLA 2).

Se han descrito otros anticuerpos que sugieren compromiso renal, muscular y pulmonar. Los anticuerpos anti-ARN polimerasa indican compromiso renal, los anticuerpos PM/Scl, compromiso muscular, y los anticuerpos antifibrilina-1, fibrosis pulmonar ${ }^{40-42}$.

También, se observa elevación de la velocidad de sedimentación globular (VSG) y la proteína C reactiva (PCR), así como la presencia de factor reumatoideo IgM en un tercio de los pacientes ${ }^{40-42}$.

Puntuación modificada de Rodnan. La gravedad de la esclerosis de la piel puede evaluarse mediante la palpación cutánea en 17 puntos diferentes. Según la localización y la extensión de la fibrosis, se asignan puntos, así: o, normal; 1, esclerosis leve; 2, esclerosis moderada, y 3, esclerosis grave con incapacidad de flejar la piel. Si el puntaje obtenido al sumarlos es mayor de 5, indica esclerodermia. Este puntaje se puede usar para evaluar la reacción terapéutica o la progresión de la enfermedad ${ }^{2,40,45,46}$.

Durometría. Es un método factible e indoloro para medir la dureza de la piel usando unidades durométricas internacionalmente estandarizadas. Las mediciones durométricas pueden discriminar entre la piel comprometida y la sana. Su resultado se correlaciona adecuadamente con la puntuación modificada de Rodnan y con la medición del grosor de la piel por ecografía ${ }^{32}$.

Ultrasonido con ondas de $20 \mathrm{MHz}$. se puede usar para medir el engrosamiento de la piel en determinados sitios y evaluar así la progresión de la enfermedad o la reacción terapéutica ${ }^{40}$.

Estudios sistémicos. El estudio de los órganos internos se utiliza para el diagnóstico y para hacer una correcta clasificación de la esclerosis sistémica ${ }^{47}$. Inicialmente, se solicita una tomografía computadorizada (TC) de tórax para determinar el compromiso pulmonar $\mathrm{y}$, después anualmente, para hacer el seguimiento y detectar fibrosis pulmonar; además, se deben practicar pruebas de función pulmonar, como capacidad vital y difusión de monóxido de carbono (CO) ${ }^{2,40}$.

Para evaluar el compromiso cardíaco, se debe hacer un electrocardiograma y un ecocardiograma, para evaluar la presión de la arteria pulmonar, la función del ventrículo derecho y el compromiso pericárdico ${ }^{2,40}$.

La evaluación renal incluye medición de la presión arterial, creatinina sérica, nitrógeno ureico en sangre (BUN) y cistatina C sérica, depuración de creatinina en orina de 24 horas y proteinuria ${ }^{2,4^{0}}$. Cuando se sospeche compromiso muscular, se debe solicitar creatina cinasa (CK), electromiografía, resonancia magnética de hombro o de cintura pélvica y biopsia muscular ${ }^{2,40}$.

Para evaluar el compromiso gastrointestinal, se solicita manometría esofágica; si hay dolor retroesternal y reflujo gastroesofágico, se debe practicar una gastros- 


\begin{tabular}{|c|c|c|}
\hline $\begin{array}{l}\text { HALLAZGOS } \\
\text { DERMATOLÓGICOS } \\
\end{array}$ & MODALIDAD TERAPÉUTICA & DOSIS \\
\hline \multirow[t]{10}{*}{ Esclerosis cutánea } & D-penicilamina & $750 \mathrm{mg} /$ día \\
\hline & $\begin{array}{l}\text { Ciclofosfamida: en pacientes que también tengan compromiso } \\
\text { sistémico (por ejemplo, fibrosis pulmonar). }\end{array}$ & 1-2 mg/kg por día \\
\hline & $\begin{array}{l}\text { Ciclosporina: en casos seleccionados que no tengan compromiso } \\
\text { renal. }\end{array}$ & $2,5-5 \mathrm{mg} / \mathrm{kg}$ por día \\
\hline & $\begin{array}{l}\text { Metotrexato: mejora la esclerosis cutánea en la esclerosis sistémica } \\
\text { cutánea difusa precoz. No se han establecido los efectos positivos } \\
\text { sobre otros órganos, } \\
\text { También es útil en los síndromes de supersposición. }\end{array}$ & $\begin{array}{l}15-25 \text { mg por semana, por vía oral } \\
\text { o subcutánea }\end{array}$ \\
\hline & $\begin{array}{l}\text { Azatioprina: se puede usar como mantenimiento, posterior a la } \\
\text { ciclofosfamida. }\end{array}$ & 50-200 mg por día (2 mg/kg) \\
\hline & Inmunoglobulina intravenosa & $2 \mathrm{~g} / \mathrm{kg}$ por 4 días al mes \\
\hline & $\begin{array}{l}\text { Fotoquimioterapia extracorpórea } \\
\text { en pacientes con esclerosis sistémica de reciente inicio }\end{array}$ & 2 días consecutivos cada mes \\
\hline & Fototerapia UVA-1: mejora la esclerosis cutánea. & $\begin{array}{l}\text { Dosis medias a altas de UVA-1, } 3 \\
\text { veces por semana }\end{array}$ \\
\hline & Fototerapia UVB-NB & $\begin{array}{l}\text { No ha demostrado ser más eficaz } \\
\text { que la fototerapia UVA-1. }\end{array}$ \\
\hline & \multicolumn{2}{|c|}{$\begin{array}{l}\text { Otros tratamientos descritos son: retinoides sistémicos combinados con otros tratamientos, } 5 \mathrm{FU} \text {, factor } \\
\text { XIII, mesilato de imatinib y rituximab. Sin embargo, se requieren más estudios clínicos para comprobar su } \\
\text { efectividad. }\end{array}$} \\
\hline \multirow{11}{*}{$\begin{array}{l}\text { Fenómeno de Raynaud } \\
\text { y úlceras digitales }\end{array}$} & \multicolumn{2}{|l|}{ Primera línea } \\
\hline & $\begin{array}{l}\text { Bloqueadores de canales de calcio (nifedipino): para el tratamiento } \\
\text { del fenómeno de Raynaud y para la prevención de úlceras digitales }\end{array}$ & 15-6o mg al día \\
\hline & $\begin{array}{l}\text { Análogos de prostaciclinas (iloprostol): para el tratamiento de } \\
\text { úlceras digitales y fenómeno de Raynaud grave }\end{array}$ & $\begin{array}{l}0,5-2 \mathrm{mg} / \mathrm{kg} / \mathrm{min} \text { por } 5-8 \text { horas por } \\
3 \text { a } 5 \text { días }\end{array}$ \\
\hline & \multicolumn{2}{|l|}{ Segunda línea } \\
\hline & $\begin{array}{l}\text { Antagonista del receptor de endotelina (bosentan): como } \\
\text { profiláctico para las úlceras digitales }\end{array}$ & $\begin{array}{l}62,5 \mathrm{mg}, 2 \text { veces al día por } 4 \\
\text { semanas; después, } 125 \mathrm{mg}, 2 \\
\text { veces al día por } 20 \text { semanas }\end{array}$ \\
\hline & \multicolumn{2}{|l|}{ Tercera línea } \\
\hline & $\begin{array}{l}\text { IECA (captopril, enalapril): mejoran el fenómeno de Raynaud y las } \\
\text { úlceras digitales }\end{array}$ & $12,5-100 \mathrm{mg} / \mathrm{día}$ \\
\hline & $\begin{array}{l}\text { Antagonista de los receptores de la angiotensina II (losartan): } \\
\text { mejoran el fenómeno de Raynaud y las úlceras digitales }\end{array}$ & $50 \mathrm{mg} / \mathrm{día}$ \\
\hline & $\begin{array}{l}\text { Inhibidores de la } 5 \text {-fosfodiesterasa (sildenafil): mejoran el } \\
\text { fenómeno de Raynaud. }\end{array}$ & $\begin{array}{l}50 \mathrm{mg}, 2 \text { veces al día por } 4 \\
\text { semanas }\end{array}$ \\
\hline & $\begin{array}{l}\text { Otros tratamientos: pentoxifilina para las úlceras digitales y nitratos } \\
\text { para el fenómeno de Raynaud }\end{array}$ & 400-1.200 mg/día \\
\hline & Cirugía de nueva irrigación & \\
\hline $\begin{array}{l}\text { Cambios en la } \\
\text { pigmentación cutánea }\end{array}$ & Análogos de la vitamina D: calcitriol & $\begin{array}{l}0,75 \mu \mathrm{g} / \text { día por } 6 \text { meses seguido } \\
\text { de } 1,25 \mu \mathrm{g} / \text { día por } 3 \text { meses }\end{array}$ \\
\hline \multirow[t]{6}{*}{ Calcinosis } & Warfarina: para calcinosis recientes & $1 \mathrm{mg} / \mathrm{día}$ \\
\hline & Minociclina & 50-100 mg/día \\
\hline & Diltiazem & $240-480 \mathrm{mg} / \mathrm{día}$ \\
\hline & Inmunoglobulina intravenosa & $2 \mathrm{~g} / \mathrm{kg}$ por 4 días al mes \\
\hline & \multicolumn{2}{|c|}{$\begin{array}{l}\text { Otros tratamientos: hidróxido de aluminio ( } 15 \mathrm{ml} 3 \text { veces al día) combinado con otros tratamientos, } \\
\text { colchicina y bifosfonatos (etidronato, } 800 \mathrm{mg} / \text { día por } 3 \text { meses cada } 6 \text { meses) }\end{array}$} \\
\hline & \multicolumn{2}{|c|}{$\begin{array}{l}\text { En pacientes resistentes a otros tratamientos: láser de } \mathrm{CO}_{2} \text {, litotripsia extracorpórea o resección } \\
\text { quirúrgica }\end{array}$} \\
\hline Telangiectasias & \multicolumn{2}{|l|}{ Láser decolorante pulsado e IPL (luz intensa pulsada) } \\
\hline
\end{tabular}

TABLA 3. Opciones terapéuticas en los hallazgos dermatológicos en la esclerosis sistémica ${ }^{16,26,38,46-49}$. 
copia para excluir esofagitis por reflujo, varices esofágicas y esófago de Barret' ${ }^{2,40}$.

\section{DIAGNÓSTICO DIFERENCIAL}

Entre los diagnósticos diferenciales se deben tener en cuenta:

- Morfea generalizada.

- Trastornos infiltrativos: amiloidosis, escleromixedema (sindrome de Arndt-Gottron) y esclerodermia de Buschke.

- Trastornos metabólicos: mixedema y porfiria cutánea tardía.

- Entidades inflamatorias: fascitis eosinofílica (síndrome de Shulman), enfermedad crónica injerto contra huésped, sarcoidosis y dermopatía fibrosante nefrógenica asociada a gadolinio.

- Otros trastornos inmunitarios: lupus eritematoso sistémico, enfermedad reumatoidea, dermatomiosistis y poliomiositis.

- Se debe, también, hacer diagnóstico diferencial con sindromes de superposición con otras enfermedades autoinmunitarias ${ }^{2,35}$.

\section{TRATAMIENTO}

El tratamiento de las manifestaciones cutáneas depende del subtipo y la fase de la enfermedad (TABLA 3).

\section{PRONÓSTICO}

La piel es un marcador de la seriedad y del pronóstico de la enfermedad. Las principales diferencias entre la esclerosis sistémica cutánea limitada y la difusa son la velocidad de progresión de la enfermedad, la extensión y la gravedad del compromiso cutáneo, y las del compromiso visceral ${ }^{24,50}$.

La progresión de la enfermedad es muy variable y difícil de predecir. Si los pacientes tienen esclerosis sistémica cutánea difusa, en los tres primeros años pueden estar en una fase inflamatoria; usualmente, durante este período la esclerosis cutánea progresa más. El tiempo que sigue a esta fase se caracteriza por la estabilización de la esclerosis o, incluso, por mejoría subjetiva; la terapia física y el ejercicio regular pueden ayudar al paciente a mantener o, incluso, a recuperar la movilidad. Estos primeros tres años son los más críticos para el desarrollo de complicaciones viscerales ${ }^{23}$.
Si no se ha presentado compromiso visceral durante los primeros seis años después de los síntomas iniciales, la probabilidad de desarrollarlo es muy baja; sólo el $10 \%$ de todos los pacientes muestran deterioro a partir de este punto ${ }^{23}$.

La tasa de mortalidad depende del grupo de pacientes y del compromiso orgánico: en la esclerosis sistémica cutánea difusa es cinco a ocho veces superior a la de la población general y es dos veces mayor que en la esclerosis sistémica cutánea limitada. En esta última, la presencia de anticuerpos anti-Th/To empeora el pronóstico ${ }^{2}$.

La combinación de varios factores de riesgo, como un puntaje alto de esclerosis cutánea, compromiso pulmonar, síntomas inflamatorios y anemia, resulta en una mayor mortalidad. Esta última es variable y corresponde al $2 \%$ si no está asociada a ningún factor de riesgo, a $26 \%$ se asocia con dos factores de riesgo y hasta de $75 \%$ si se asocian cuatro factores de riesgo ${ }^{23}$.

La supervivencia a 15 años en la esclerosis sistémica cutánea difusa es del $50 \%$, mientras que, en la limitada, es del $70 \%$. De todas las muertes por causas relacionadas con la esclerosis sistémica, en el $50 \%$ se trata de compromiso pulmonar; de estas, el $50 \%$ son por hipertensión pulmonar y el $25 \%$ son por fibrosis pulmonar. Los pacientes con compromiso cardíaco tienen peor pronóstico ${ }^{2,51,52}$.

\section{CONCLUSIÓN}

La esclerosis sistémica es una enfermedad multisistémica del tejido conjuntivo, en la cual el compromiso cutáneo es un marcador de la gravedad y del pronóstico de la enfermedad. Por lo anterior, es importante conocer el espectro clínico de las manifestaciones cutáneas, con el fin de hacer un diagnóstico certero y temprano, e instaurar un tratamiento adecuado y oportuno que pueda modificar el curso de la enfermedad, mejorando la calidad de vida de los pacientes.

\section{REFERENCIAS}

1. Balbir-Gurman A, Baun-Moscovici Y. Scleroderma - New aspects in pathogenesis and treatment. Best Pract Res Clin Rheumatol. 2012;26:13-24.

2. Unanue L, González MR, Gardeazábal J. Esclerodermia (sclerosis sistémica). Piel. 2010;25:252-66.

3. Katsumoto TR, Whitfield ML, Connolly MK. The pathogenesis of systemic sclerosis. Annu Rev Pathol. 2011;6:509-37.

4. Szekanecz Z, Koch AE. Endothelial cells in inflammation and angiogenesis. Curr Drug Targets. 2005;4:319-23.

5. Sticherling M. Systemic sclerosis - dermatological aspects. 
Part 1: Pathogenesis, epidemiology, clinical findings. J Dtsch Dermatol Ges. 2012;10:705-16.

6. Mayes MD. Scleroderma epidemiology. Rheum Dis Clin North Am. 2003;29:239-54.

7. Chifflot H, Fautzi B, Sordet C, Chatelus E, Sibilia J. Incidence and prevalence of systemic sclerosis: A systematic literature review. Semin Arthritis Reum. 2008;37:223-35.

8. Laing TJ, Gillespie BW, Toth MB, Mayes MD, Gallavan Jr RH, Burns CJ, et al. Racial differences in scleroderma among women in Michigan. Arthritis Rheum. 1997;40:734-42.

9. Vancheeswaran R, Black CM, David J, Hasson N, Harper J, Atherton D, et al. Childhood-onset scleroderma: Is it different from adult-onset disease. Arthritis Rheum. 1996;39:1041-9.

10. Scalapino K, Arkachaisri T, Lucas M, Fertig N, Helfrich DJ, Londino Jr AV, et al. Childhood onset systemic sclerosis: Classification, clinical and serologic features, and survival in comparison with adult onset disease. J Rheumatol. 2006;33:1004-13.

11. Janet E. Pope, Sindhu R. Johnson. New Classification Criteria for Systemic Sclerosis (Scleroderma). Rheum Dis Clin N Am 41. 2015; 383-98.

12. LeRoy EC, Medsger Jr TA. Criteria for the classification of early systemic sclerosis. J Rheumatol. 2001;28:1573.

13. Poormoghim H, Lucas M, Fertig N, Medsger Jr TA. Systemic sclerosis sine scleroderma: Demographic, clinical and serologic features and survival in forty-eight patients. Arthritis Rheum. 2000;43:444-51.

14. Arnson Y, Amital H, Guiducci S, Matucci-Cerinic M, Valentini $\mathrm{G}$, Barzilai $\mathrm{O}$, et al. The role of infections in the immunopathogenesis of systemic sclerosis evidence from serological studies. Ann N Y Acad Sci. 2009;1173:627-32.

15. Mora GF. Systemic sclerosis: Environmental factors. J Rheumatol. 2009;36:2383-96.

16. Viswanath V, Phiske M, Gopalani V. Systemic sclerosis: Current concepts in pathogenesis and therapeutic aspects of dermatology manifestations. Indian J Dermatol. 2013;58:255-69.

17. Mahoney WM Jr, Fleming JN, Schwartz SM. A unifying hypothesis for scleroderma: identifying a target cell for scleroderma. Curr Rheumatol Rep. 2011;13:28-36.

18. Wigley FM. Clinical practice. Raynaud's phenomenon. N Engl J Med. 2002;347:1001-8.

19. Tolosa C, Simeon C, Gabarro L. El fenómeno de Raynaud. Med Clin. 2009;132:712-8.

20. Nietsche A. Raynaud, úlceras digitales y calcinosis en esclerodermia. Reumatol Clinic. 2012;8:270-7.

21. Strange G, Nash P. The manifestation of vasculophaty in systemic sclerosis and its evidence based therapy. Int J Rheum Dis. 2009;12:192-206.

22. Jones S. Skin manifestations of systemic disease. Medicine. 2004;32:40-3.

23. Chung L, Lin J, Furst D, Fiorentino D. Systemic and localizated scleroderma. Clin Dermatol. 2006;24:374-92.

24. Krieg T, Takehara K. Skin disease: A cardinal feature of systemic sclerosis. Rheumatology. 2009;48:14-8.

25. Steen V, Denton CP, Pope JE, Matucci-Cerinic M. Digital ulcers: Overt vascular disease in systemic sclerosis. Rheumatology (Oxford). 2009;4(Suppl.3):19-24.

26. Arenas C, Jiménez J, González MI. Úlcera digital como mani- festación inicial de esclerodermia sistémica cutánea límitada. Rev Asoc Colomb Dermatol.2013;21:360-3.

27. Amanzi L, Braschi F, Fiori G, Galluccio F, Miniati I, Giudicci $\mathrm{S}$, et al. Digital ulcers in scleroderma: Staging, characteristics and sub-setting through observation of 1614 lesions. Rheumatology. 2010;49:1374-82.

28. Alivernini S, De Santis M, Tolusso B, Mannocci A, Bosello SL, Peluso G, et al. Skin ulcers in systemic sclerosis: Determinants of presence and predictive factors of healing. J Am Acad Dermatol. 2009;60:426-35.

29. Tabata H, Hara N, Otsuka S, Yamakage A, Yamazaki S, Koibuchi N. Correlation between diffuse pigmentation and keratinocyte derived endothelin-1 in systemic sclerosis. Int J Dermatol. 2000;39:899-902.

30. Boulman N, Slobodin G, Rozenbaum M, Rosner I. Calcinosis in rheumatic diseases. Semin Artritis Rheum. 2005;34:805-12.

31. Davies CA, Jeziorska M, Freemont AJ, Herrick AL. Expression of osteonectin and matrix Gla protein in scleroderma patients with and without calcinosis. Rheumatology.2006;45:1349-55.

32. Czirjak L, Foeldvari I, Muller-Ladner U. Skin Involvement in systemic sclerosis. Rheumatology. 2008;47:44-5.

33. Pope JE. Musculoskeletal involvement in scleroderma. Rheum Dis Clin North Am.2003;29:391-408.

34. Clements PJ, Furst DE, Campion DS, Bohan A, Harris R, Levy J, Paulus HE. Muscle disease in progressive systemic sclerosis: Diagnostic and therapeutic considerations. Arthritis Rheum.1978;21:62-71.

35. Denton C, Black C. Scleroderma (Systemic sclerosis). In: Wolff K, Goldsmith LA, Katz SI, Gilchrest BA, Paller AS, Leffell DJ, editors. Fitzpatrick's Dermatology in General Medicine. Seventh edition. New York: McGraw-Hill; 2008. p. 1553-62.

36. Chojnowski MM, Felis-Giemza A, Olesinska M. Capillaroscopy-a role in modern rheumatology. Rheum.2016;54:67-72.

37. Cutulo M, Sulli A, Pizzorni C, Accardo S. Nailfold videocapillaroscopy assesment of microvascular damage in systemic sclerosis. J Rheumatol. 2000;27:155-160.

38. Sebastiani M, Manfredi A, Colaci M, D’amico R, Malagoli V, Giuggioli D, Ferri C. Capillaroscopic skin ulcer risk index: A new prognostic tool for digital skin ulcer development in systemic sclerosis patients. Arthritis Rheum.2009;61:688-94.

39. Sebastiani M, Manfredi A, Vukatana G, Moscatelli S, Riato L, Bocci M,et al. Predictive role of capillaroscopic skin ulcer risk index in systemic sclerosis: A multicenter validation study. Ann Rheum Dis.2012;71:67-70.

40. Sticherling M. Systemic sclerosis - focus on dermatological aspects. Part 2: Diagnostics, therapy. J Dtsch Dermatol Ges. 2012;10:783-91.

41. Klein-Weigel P, Opitz C, Riemekasten G. Systemic sclerosis: A systematic overview. Part 1. Disease characteristics and classification, pathophysiologic concepts, and recommendations for diagnosis and surveillance. Vasa. 2011;40:6-19.

42. Grassegger A, Pohla-Gubo G, Frauscher M, Hintner H. Autoantibodies in systemic sclerosis (scleroderma): Clues for clinical evaluation, prognosis and pathogenesis. Wien Med Wochenschr. 2008;158:19-28.

43. 43. Ho KT, Reveille JD. The clinical relevance of autoantibodies in scleroderma. Arthritis Res Ther. 2003;5:80-93

44. Czömpóly T, Simon D, Czirják L, Németh P. Anti-topoisomerasa I autoantibodies in systemic sclerosis. Autoimmun Rev. 2009;8:692-6. 
45. Black CM. Measurement of skin involvement in scleroderma. J Rheumatol. 1995;22:1217-9.

46. Amjadi S, Maranian P, Furst DE, Clements PJ, Wong WK, Postlethwaite AE, et al. Course of the modified Rodnan skin thickness score in systemic sclerosis clinical trials: Analysis of three large multicenter, double-blind, randomized con- trolled trials. Arthritis Rheum. 2009;60:2490-8.

47. Walker UA, Tyndall A, Czirjak L, Denton C, Farge-Bancel D, Kowal-Bielecka O, et al. Clinical risk assessment of organ manifestations in systemic sclerosis: A report from the EULAR Scleroderma Trials and Research group database. Ann Rheum Dis. 2007;66:754-63.

48. Vitiello M, Abuchar A, Santana N, Dehesa L, Kerdel FA. An update on the treatment of the cutaneous manifestation of systemic sclerosis: The dermatologist's point of view. J Clin Aesthet Dermatol. 2012;5:33-43.

49. Kowal-Bieleka O, Landewe R, Avouac J, Chwiesko S, Miniati $\mathrm{I}$, Czirjak L, et al. EULAR recommendations for the treatment of systemic sclerosis: A report from the EULAR Scleroderma Trials and Research group (EUSTAR). Ann Rheum Dis. 2009;68:620-8.

50. Meyer O. Prognostic markers for systemic sclerosis. Joint Bone Spine. 2006;73:490-4.

51. Steen VD. The lung in systemic sclerosis. J Clin Rheumatol. 2005;11:40-6.

52. Ioannidis JP, Vlachoyiannopoulos PG, Haidich AB, Medsger Jr TA, Lucas M, Michet CJ, et al. Mortality in systemic sclerosis: An international meta-analysis of individual patient data. Am J Med. 2005;118:2-10. 